\title{
Densidade e tamanho de formigueiros de Acromyrmex crassispinus em plantios de Pinus taeda
}

\author{
Mariane Aparecida Nickele(1), Wilson Reis Filho ${ }^{(2)}$, Edilson Batista de Oliveira(2) e Edson Tadeu lede ${ }^{(2)}$ \\ (1) Universidade Federal do Paraná, Departamento de Zoologia, Caixa Postal 319, CEP 83411-000 Colombo, PR. \\ E-mail: nickele.mariane@gmail.com (2)Embrapa Florestas, Estrada da Ribeira, Caixa Postal 319, CEP 83411-000 Colombo, PR. \\ E-mail: wilson@cnpf.embrapa.br, edilson@cnpf.embrapa.br, iedeet@cnpf.embrapa.br
}

\begin{abstract}
Resumo - O objetivo deste trabalho foi avaliar a densidade e o tamanho dos formigueiros de Acromyrmex crassispinus, ao longo do ano, em plantios de Pinus taeda de diferentes idades. Os experimentos foram realizados, em Rio Negrinho e em Três Barras, SC, em talhões de P. taeda com diferentes idades (recém-plantado, três anos e seis anos de idade). Em cada tratamento, foram demarcadas três parcelas de 1 ha. Os formigueiros foram classificados em três classes de tamanho: classe I, até $30 \mathrm{~cm}$ de diâmetro; classe II, 31 a $60 \mathrm{~cm}$; e classe III, mais que $61 \mathrm{~cm}$. A densidade de formigueiros de $A$. crassispinus foi menor no início do desenvolvimento da floresta, praticamente duplicou nos plantios com três anos, e reduziu quando a floresta estava com seis anos. A maior densidade de formigueiros ocorreu no período de dezembro a abril. Os formigueiros aumentaram de tamanho com a idade do pínus
\end{abstract}

Termos para indexação: Formicidae, formigas cortadeiras, praga florestal, reflorestamentos.

\section{Density and size of Acromyrmex crassispinus nests in Pinus taeda plantations}

\begin{abstract}
The objective of this work was to evaluate the density and size of Acromyrmex crassispinus nests in Pinus taeda plantations along the year. The experiments were carried out in Rio Negrinho and Três Barras, SC, Brazil, in P. taeda plantations of different ages (recently planted and three and six years old). Three plots of one hectare were delimited in each treatment. The nests were classified according to three size classes: class I, up to $30 \mathrm{~cm}$ diameter; class II, 31 up to $60 \mathrm{~cm}$; class III, above $61 \mathrm{~cm}$. The density of $A$. crassispinus nests was low in recently planted areas, nearly twofold in three year-old plantations, and it reduced when the forest was six years old. The greatest nest density occurred in the period from December to April. The nests size increased along with the age of the $P$. taeda plantations.
\end{abstract}

Index terms: Formicidae, leaf-cutting ants, forest pests, reforestation.

\section{Introdução}

A produção e o aproveitamento da madeira são importantes, pois existe uma crescente demanda do produto como matéria-prima em vários segmentos produtivos. As plantações comerciais visam a suprir essa demanda. A área cultivada com espécies florestais, no Brasil, corresponde a aproximadamente 5,6 milhões de hectares, sendo 3,4 milhões de hectares com Eucalyptus spp., 1,8 milhão de hectares com Pinus spp. e 326 mil hectares com outras espécies. No Sul do país, cerca de $80 \%$ das florestas plantadas são constituídas de Pinus taeda L., devido à sua alta produtividade e qualidade de matéria-prima (Sociedade Brasileira de Silvicultura, 2006).

Entre os problemas silviculturais dessas florestas implantadas, sobressaem as formigas cortadeiras dos gêneros Atta (saúvas) e Acromyrmex (quenquéns), que alcançaram o nível de praga pela magnitude dos prejuízos que causam aos plantios florestais.

As saúvas e as quenquéns usam exclusivamente substratos vegetais para o cultivo do fungo que utilizam na alimentação, destacando-se como formigas cortadeiras de importância econômica. São nocivas ao sistema agroflorestal, pois podem cortar e utilizar ampla faixa de espécies vegetais que são cultivadas pelo homem (Lima et al., 2001).

Link et al. (2001) verificaram que o potencial médio de dano de Acromyrmex crassispinus (Forel) foi de oito mudas de Pinus com cerca de três meses de idade por intervalo de três dias de inspeção em áreas onde foi realizado um controle pré-plantio em Vargem Bonita, SC. Cantarelli (2005) verificou que $20,8 \%$ do total de 
mudas recém-plantadas de $P$. taeda foram atacadas por A. heyeri Forel e A. lobicornis Emery 65 dias após o plantio, na Argentina, mas houve redução na taxa de herbivoria com o passar do tempo.

A determinação da densidade de formigueiros em áreas cultivadas é fundamental, pois permite fazer inferências sobre o potencial de danos das espécies e, consequentemente, sobre o combate racional desses insetos (Pereira et al., 1999).

A espécie $A$. crassispinus é a formiga cortadeira mais comum na Região Sul do Brasil (Gonçalves, 1961; Rando \& Forti, 2005) e nos plantios de P. taeda localizados no norte de Santa Catarina. No entanto, são poucas as pesquisas feitas sobre essa espécie.

Este trabalho teve como objetivo determinar a densidade e o tamanho dos formigueiros de A. crassispinus, ao longo do ano, em plantios de $P$. taeda de diferentes idades.

\section{Material e Métodos}

As áreas experimentais estavam localizadas em plantios de P. taeda nos municípios de Rio Negrinho (2615'16"S; 49³1'6"W; altitude de $790 \mathrm{~m}$ ), de propriedade da empresa Battistella Florestal, e Três Barras (266'23"S; 50¹9'20"W; altitude de $802 \mathrm{~m}$ ), de propriedade da empresa Rigesa, ambos localizados no Planalto Norte Catarinense.

O plantio de $P$. taeda em Rio Negrinho é para a produção de madeira livre de nós, por isso é realizada a poda de galhos inferiores a partir do terceiro ano, o que agrega maior valor à madeira. Em Três Barras, o plantio é para a produção de papel e celulose, por isso não é realizada a poda de galhos. Em ambos os municípios, o combate às formigas cortadeiras é realizado de maneira sistemática antes do plantio e de maneira localizada após o plantio, principalmente aos 15 e 30 dias após o plantio. A manutenção do sub-bosque é realizada com aplicação de herbicida somente na linha de plantio ou com roçadas ao longo da linha de plantio.

Os experimentos foram realizados em plantios de P. taeda com diferentes idades em áreas de reforma, ou seja, áreas em que havia Pinus antes do novo plantio. Cada idade de plantio correspondeu a um tratamento nos dois locais, classificado da seguinte maneira: T1, área de $P$. taeda recém-plantado; T2, área de $P$. taeda com três anos; T3, área de P. taeda com seis anos. O tipo de solo das áreas e seus atributos estão apresentados na Tabela 1. Para cada tratamento, em cada local, foram delimitadas três parcelas de 1 ha $(100 \times 100 \mathrm{~m})$. $\mathrm{O}$ espaçamento entre as plantas era de $2,5 \times 2,5 \mathrm{~m}$ (1.600 plantas $\mathrm{ha}^{-1}$ ), exceto na área com pínus recém-plantado de Rio Negrinho, onde o espaçamento era de $2 \times 2,5 \mathrm{~m}$ ( 2.000 plantas ha $\left.^{-1}\right)$. Não foi realizado o combate às formigas cortadeiras no T1. Já em T2 e T3, o combate às formigas cortadeiras foi realizado somente no primeiro ano de plantio. Foi realizada a contagem do número (densidade) de formigueiros de A. crassispinus na área total de cada parcela em cada um dos tratamentos. No T1, as avaliações foram realizadas mensalmente nos primeiros 6 meses e aos $9 \mathrm{e}$ 12 meses após o plantio, com início em março de 2007, em Rio Negrinho, e em abril de 2007, em Três Barras. Nos tratamentos T2 e T3, foram realizadas avaliações em março, junho, setembro e dezembro de 2007, nos dois locais. Foi efetuada análise de variância (ANOVA) do número de formigueiros e as médias foram comparadas pelo teste de Tukey, a 5\% de probabilidade.

Tabela 1. Caracterização dos solos nas áreas de Pinus taeda recém-plantado, com três anos e com seis anos de idade, em Rio Negrinho e Três Barras, SC.

\begin{tabular}{|c|c|c|c|c|c|}
\hline Tratamentos & Tipo de solo & Horizonte & Argila $\left(\mathrm{g} \mathrm{kg}^{-1}\right)$ & $\mathrm{pH}\left(\mathrm{H}_{2} \mathrm{O}\right)$ & $\mathrm{MO}\left(\mathrm{g} \mathrm{kg}^{-1}\right)$ \\
\hline \multirow{3}{*}{ Recém-plantado } & \multirow{3}{*}{ Cambissolo Húmico alumínico típico } & Rio Negri & & & \\
\hline & & Ap $(0-20 \mathrm{~cm})$ & 310 & 3,9 & 32 \\
\hline & & $\mathrm{BA}(30-40 \mathrm{~cm})$ & 330 & 4,1 & 30 \\
\hline \multirow[t]{2}{*}{ Três anos } & \multirow[t]{2}{*}{ Cambissolo Húmico alumínico saprolítico } & Ap $(0-20 \mathrm{~cm})$ & 220 & 3,6 & 44 \\
\hline & & $\mathrm{Bi}(30-50 \mathrm{~cm})$ & 320 & 3,7 & 27 \\
\hline \multirow[t]{2}{*}{ Seis anos } & \multirow[t]{2}{*}{ Neossolo Regolítico alumínico húmico } & $\mathrm{A}(0-20 \mathrm{~cm})$ & 570 & 4,3 & 32 \\
\hline & & $\mathrm{B}(50-70 \mathrm{~cm})$ & 620 & 4,4 & 26 \\
\hline \multirow{3}{*}{ Recém-plantado } & \multirow{3}{*}{ Cambissolo Háplico alumínico argissólico } & Três Barr & & & \\
\hline & & $\mathrm{A}(0-19 \mathrm{~cm})$ & 680 & 4,9 & 47 \\
\hline & & $\mathrm{A} 2(19-38 \mathrm{~cm})$ & 720 & 4,6 & 39 \\
\hline \multirow[t]{2}{*}{ Três anos } & \multirow[t]{2}{*}{ Nitossolo Vermelho distrófico típico } & $\mathrm{A}(0-18 \mathrm{~cm})$ & 480 & 4,6 & 63 \\
\hline & & $\mathrm{A} 2(18-67 \mathrm{~cm})$ & 520 & 4,8 & 33 \\
\hline \multirow[t]{2}{*}{ Seis anos } & \multirow[t]{2}{*}{ Argissolo Bruno-Acinzentado alítico típico } & Ap $(0-14 \mathrm{~cm})$ & 440 & 4,7 & 26 \\
\hline & & $\mathrm{A} 2(14-54 \mathrm{~cm})$ & 460 & 4,5 & 18 \\
\hline
\end{tabular}


Todos os formigueiros foram medidos registrandose o maior comprimento e a maior largura, embora o comprimento do eixo maior tenha sido considerado como o diâmetro aproximado do ninho (Link et al., 2001), e foram categorizados em três classes de tamanho: classe I, com até $30 \mathrm{~cm}$ de diâmetro; classe II, entre 31 e $60 \mathrm{~cm}$; e classe III, com mais de $61 \mathrm{~cm}$. Para o cálculo da frequência de formigueiros nas diferentes classes de tamanho, os formigueiros que estavam presentes em mais de uma avaliação foram contabilizados apenas uma vez.

Foram coletadas dez operárias máximas de cada formigueiro para identificação da espécie. Todo o material foi acondicionado em frascos devidamente etiquetados, com álcool $70 \%$. As formigas coletadas foram levadas ao Laboratório de Entomologia da Embrapa Florestas, montadas e identificadas com auxílio de chaves de identificação propostas por Gonçalves (1961), Mayhé-Nunes (1991) e Forti et al. (2006). Para a confirmação da espécie de formiga cortadeira, alguns exemplares foram encaminhados ao Laboratório de Insetos Sociais da Faculdade de Ciências Agronômicas da Unesp, em Botucatu, SP.

\section{Resultados e Discussão}

Além da espécie $A$. crassispinus, que foi a mais frequente $(95,83 \%)$ nos plantios de $P$. taeda em Rio Negrinho, constatou-se a ocorrência das espécies Acromyrmex subterraneus subterraneus Forel (2,78\%) e Acromyrmex aspersus (F. Smith) (1,39\%).

Em Três Barras, A. crassispinus também foi a espécie que predominou $(95,23 \%)$, e foi constatada a presença de Acromyrmex rugosus rugosus (F. Smith) (3,57\%) e A. subterraneus subterraneus $(1,19 \%)$.

A espécie $A$. crassispinus é a formiga cortadeira mais comum na Região Sul do Brasil (Gonçalves, 1961; Rando \& Forti, 2005). É uma espécie com importância econômica em reflorestamentos de P. taeda (Lopes et al., 2004). É considerada uma das quenquéns mais abundantes e é causadora de elevados prejuízos, tanto pela frequência de ninhos em algumas regiões quanto pelo número de plantas atacadas pela espécie (Link et al., 2000).

As maiores densidades de formigueiros de A.crassispinus foram registradas nos meses de dezembro a abril em ambos os municípios (Figuras 1 e 2). Reis Filho \& Oliveira (2002) verificaram que o período de maior ocorrência dessa espécie de formiga cortadeira em plantios de P. taeda, no município de Três Barras, foi de dezembro a abril, sendo crítico nos meses de fevereiro, março e abril. Esses mesmos autores constataram a presença de reprodutores no interior dos formigueiros no mês de agosto, o que sugere que a revoada de A. crassispinus ocorre durante a primavera. $\mathrm{O}$ aumento na densidade de formigueiros verificado nos meses de fevereiro, março e abril ocorreu provavelmente devido à instalação de novos formigueiros oriundos da última revoada.

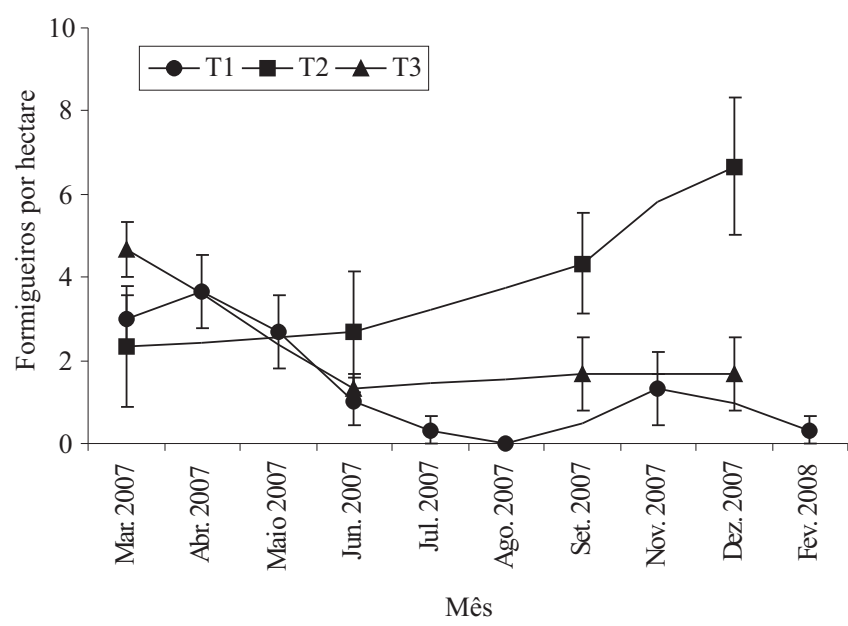

Figura 1. Densidade de formigueiros de Acromyrmex crassispinus (média \pm erro-padrão da média) em plantios de Pinus taeda. T1, pínus recém-plantado; T2, pínus com três anos; T3, pínus com seis anos de idade, em Rio Negrinho, SC.

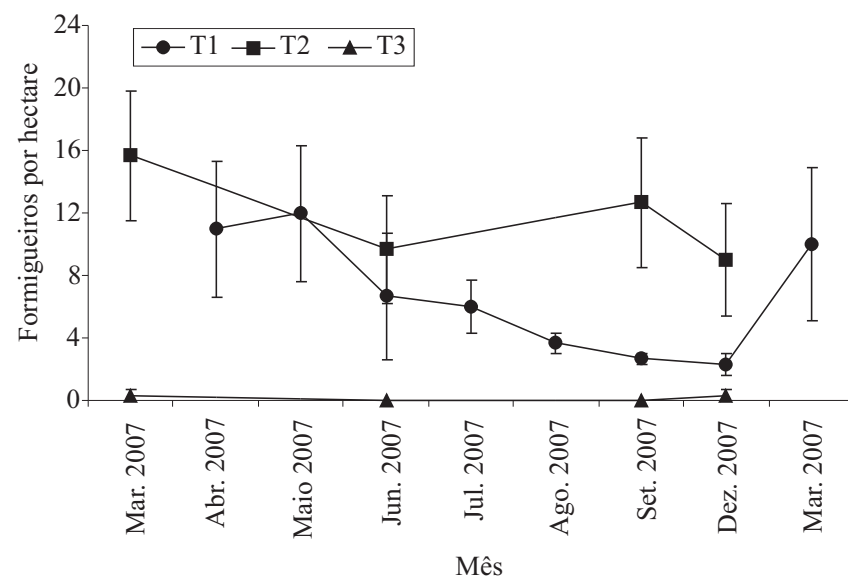

Figura 2. Densidade de formigueiros de Acromyrmex crassispinus (média \pm erro-padrão da média) em plantios de Pinus taeda. T1, pínus recém-plantado; T2, pínus com três anos; T3, pínus com seis anos de idade, em Três Barras, SC. 
Constatou-se que $A$. crassispinus transferem periodicamente os seus formigueiros. Tal comportamento também já foi observado por Link et al. (2001), em plantios de Pinus e Eucalyptus, no Município de Vargem Bonita, SC, embora a distância em que os formigueiros transferem-se ainda seja desconhecida. A espécie A. crassispinus tem essa possibilidade de mudar de local, pois seus ninhos são superficiais e têm uma única câmara grande situada em uma escavação rasa. Os ninhos são inteiramente cobertos por folhas secas e resíduos vegetais que envolvem a cultura de fungo.

Foi registrado, em média, 1,54 formigueiro de A. crassispinus por hectare durante todo o período de avaliação na área de Rio Negrinho com pínus recém-plantado. A maior concentração $(70,59 \%)$ de formigueiros constatada foi da classe de tamanho I (Tabela 2 e Figura 3). Na área com três anos, foram encontrados, em média, 4,08 formigueiros por hectare, com predominância de tamanhos médio $(38,71 \%)$ e

Tabela 2. Densidade (média \pm erro-padrão da média) de formigueiros de Acromyrmex crassispinus, em áreas de Pinus taeda recém-plantado, com três anos e com seis anos de idade, em Rio Negrinho e Três Barras, $\mathrm{SC}^{(1)}$.

\begin{tabular}{lcr}
\hline Tratamentos & \multicolumn{2}{c}{ Densidade de formigueiros } \\
\cline { 2 - 3 } & Rio Negrinho & Três Barras \\
\hline Recém-plantado & $1,54 \mathrm{a} \pm 0,33$ & $6,83 \mathrm{a} \pm 1,34$ \\
Três anos & $4,08 \mathrm{a} \pm 1,24$ & $11,66 \mathrm{~b} \pm 1,84$ \\
Seis anos & $2,33 \mathrm{a} \pm 0,51$ & $0,17 \mathrm{c} \pm 0,11$ \\
\hline (1)
\end{tabular}

${ }^{(1)}$ Médias seguidas de letras iguais na coluna não diferem entre si pelo teste de Tukey, a $5 \%$ de probabilidade.

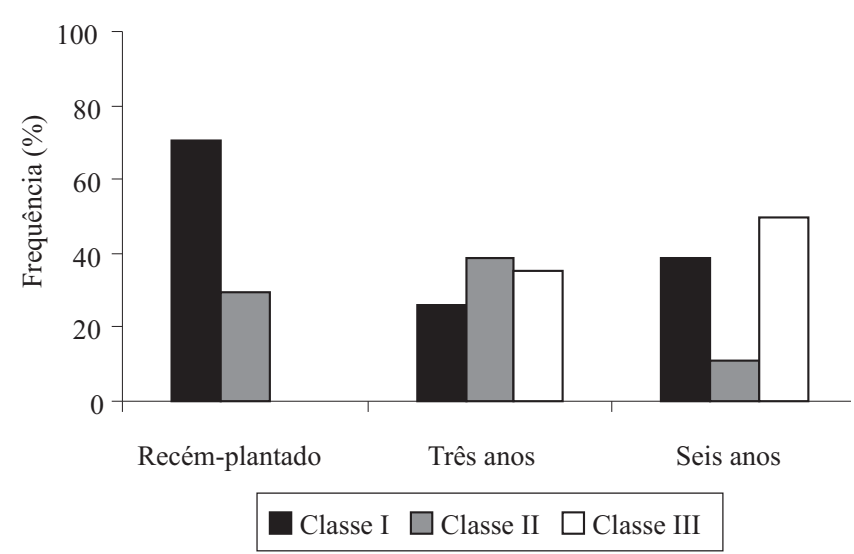

Figura 3. Frequência de formigueiros de Acromyrmex crassispinus, em áreas de Pinus taeda recém-plantado, com três anos e com seis anos de idade, por classe de tamanho: classe I, até $30 \mathrm{~cm}$ de diâmetro; classe II, entre 31 e $60 \mathrm{~cm}$ e classe III, maior que $61 \mathrm{~cm}$, em Rio Negrinho, SC. grande $(35,48 \%)$. Já na área com seis anos, a média foi de 2,33 formigueiros por hectare, com predominância da classe III $(50 \%)$. Não houve diferença significativa na densidade de formigueiros entre os tratamentos.

Devido à realização da poda dos galhos das árvores em Rio Negrinho, o dossel dos plantios com seis anos de idade não era fechado, permitindo incidência de luz solar no interior da floresta, como ocorreu nas áreas com pínus recém-plantado e de três anos. Esse fato pode ter favorecido a ocorrência dos ninhos de $A$. crassispinus em todas áreas. Clark \& Evans (1955) verificaram que Atta laevigata (F. Smith) prefere áreas com maior exposição solar. Os formigueiros de Acromyrmex lobicornis Emery encontram-se, na sua maioria, em lugares relativamente abertos e bem abaixo de árvores ou arbustos, que são relativamente ensolarados, o que pode ser uma característica dos padrões de distribuição espacial dessas espécies (Bucher \& Montenegro, 1974).

$\mathrm{Na}$ área de Três Barras com pínus recém-plantado, foi constatada uma média de 6,83 formigueiros por hectare durante todo o período de avaliação, e $79,76 \%$ concentravam-se na classe de tamanho I (Tabela 2 e Figura 4). Na área com três anos, a densidade foi, em média, de 11,66 formigueiros por hectare, e $60 \%$ dos formigueiros pertenciam à classe II. Na área com seis anos de idade, foi rara a presença de formigueiros. A média foi de 0,17 formigueiro por hectare. Essa área apresentava o dossel totalmente fechado e sem a incidência direta do sol no interior

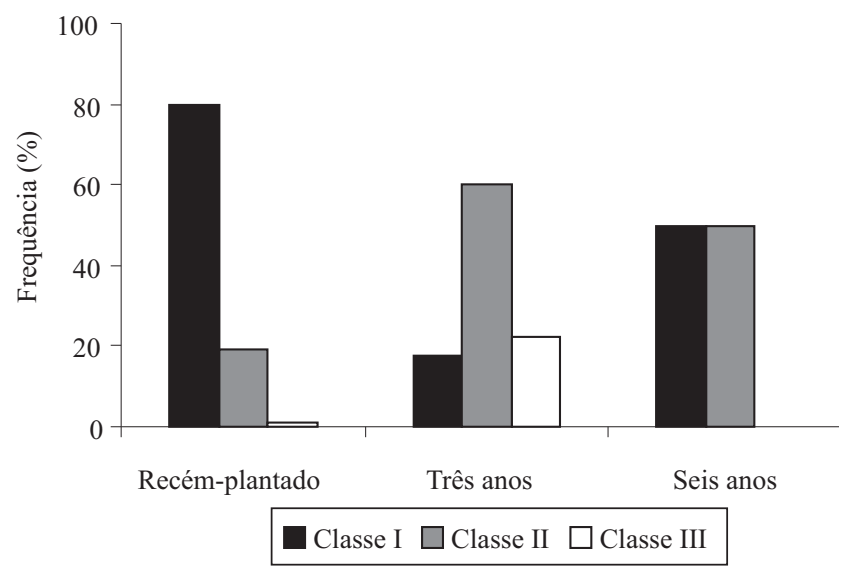

Figura 4. Frequência de formigueiros de Acromyrmex crassispinus, em áreas de Pinus taeda recém-plantado, com três anos e com seis anos de idade, por classe de tamanho. Classe I, até $30 \mathrm{~cm}$ de diâmetro; classe II, entre 31 e $60 \mathrm{~cm}$; classe III, maior que $61 \mathrm{~cm}$, em Três Barras, SC. 
da floresta devido à não realização da poda dos galhos inferiores, além da ausência de sub-bosque. Nessa situação, os únicos formigueiros de $A$. crassispinus encontrados pertenciam às classes I e II e estavam localizados próximos a pequenas clareiras. Todos os tratamentos avaliados em Três Barras apresentaram diferenças em relação à densidade de formigueiros.

A densidade de formigueiros de $A$. crassispinus foi influenciada pelo manejo florestal. Em Rio Negrinho, onde é realizada a poda dos galhos, não houve diferenças na densidade de formigueiros em plantios de P. taeda com diferentes idades. Em Três Barras, como o objetivo dos reflorestamentos é a produção de papel e celulose, a poda dos galhos não é realizada e, nesse caso, houve diferenças na densidade de formigueiros.

Outro fator que pode influenciar a densidade de formigueiros é o tipo de solo, que em Três Barras era diferente nos três tratamentos. Neste trabalho não se pôde verificar se o solo estava influenciando a densidade de formigueiros, mas Hernández \& Jaffé (1995) relataram que um dos fatores mais importantes que favorecem o estabelecimento de formigueiros de Atta laevigata em plantios de P. caribaea é o tipo de solo. Esses autores verificaram uma correlação altamente positiva entre a profundidade da camada de argila e o número de formigueiros, ou seja, quanto maior a profundidade da camada de argila, mais arenoso é o solo e maior será a probabilidade de encontrar altas populações de ninhos de Atta. Bento et al. (1991) encontraram uma relação positiva entre a densidade de sauveiros e solos de baixa fertilidade. A profundidade da câmara inicial de um sauveiro coincide com as camadas mais superficiais do solo, sendo que nessas camadas predominam os maiores números de microrganismos do solo, devido à abundância de matéria orgânica e nutrientes. As condições de solos mais pobres, tanto em termos de nutrientes como de populações microbianas naturais, propiciaram um meio mais favorável para o estabelecimento de formigueiros incipientes, pela ausência tanto de entomopatógenos quanto de possíveis antagonistas ao fungo simbionte das formigas. Diehl-Fleig \& Rocha (1998) também verificaram que os solos com baixa fertilidade foram preferidos pelas rainhas de Acromyrmex striatus Roger no ato da fundação da colônia.

As diferentes classes de tamanho dos formigueiros apresentam relação com a idade, ou seja, quanto mais velho era o formigueiro, maior era o seu tamanho.
A densidade de formigueiros da classe de tamanho I permaneceu constante em todos os tratamentos em ambos os municípios, provavelmente em razão das revoadas que ocorrem todos os anos. Houve predominância de formigueiros dessa classe nas áreas com pínus recém-plantado. Talhões recém-plantados são áreas propícias para a instalação e estabelecimento de rainhas logo após as revoadas, pois as rainhas procuram locais mais destituídos de vegetação para iniciar a construção de seu ninho (Lima et al., 2001).

Link et al. (2001) verificaram, em talhões de Pinus spp. com aproximadamente três meses de idade, maior ocorrência de formigueiros de tamanho médio de A. crassispinus $(51,52 \%)$, discordando dos resultados encontrados no presente estudo. No entanto, antes do plantio das mudas na área estudada por aqueles autores, foi realizado um combate prévio, que provavelmente foi mais eficiente no controle de formigueiros de tamanho pequeno.

Nas áreas com três anos de idade, houve predominância de formigueiros das classes II e III, ou seja, formigueiros que já estavam instalados na área há algum tempo. A menor ocorrência de formigueiros da classe I foi, provavelmente, devido à presença de grande quantidade de vegetação nativa entre as linhas de plantio nessa fase de desenvolvimento da floresta. Em solos com maior cobertura vegetal, os formigueiros iniciais têm menor taxa de sobrevivência, pois a cobertura vegetal pode dificultar o pouso e a instalação das rainhas durante a revoada, além de favorecer a atuação de seus inimigos naturais no momento da fundação do formigueiro (Lima et al., 2001).

A vegetação entre as linhas de plantio, quando não competitiva com as plantas cultivadas, deve ser mantida, pois serve de alimento e abrigo para diversas espécies de inimigos naturais, além de servir de substrato para o fungo das formigas (Araújo et al., 2003). Almeida et al. (1983) verificaram que a densidade de sauveiros em talhões de Eucalyptus foi 18 vezes menor em áreas com sub-bosque denso em comparação com áreas sem sub-bosque.

A densidade de formigueiros de $A$. crassispinus foi menor no início de desenvolvimento da floresta. Ela praticamente duplicou nos plantios com três anos de idade e sofreu uma redução quando a floresta estava com seis anos de idade (Figura 5). Isso ocorreu em ambos os municípios estudados, embora não houvesse diferenças significativas em Rio Negrinho. 


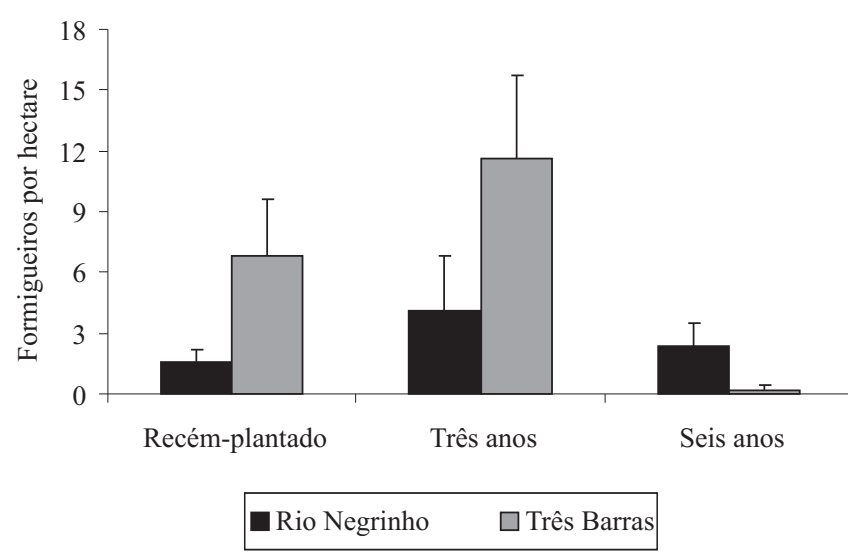

Figura 5. Densidade média e intervalo de confiança $(\mathrm{p}<0,05)$ de formigueiros de Acromyrmex crassispinus, em áreas de Pinus taeda recém-plantado, com três anos e com seis anos de idade, em Rio Negrinho e Três Barras, SC.

Zanetti et al. (2000) verificaram situação semelhante na densidade de formigueiros iniciais de Atta spp. em plantios de Eucalyptus em João Pinheiro, MG. A densidade de sauveiros foi menor no início de desenvolvimento da floresta, aumentou até os três anos e então reduziu até os níveis iniciais, permanecendo estável até a idade de corte da floresta.

A razão para a ocorrência dessa situação é que, em áreas recém-plantadas, os formigueiros novos se instalam a partir do momento em que ocorrem as revoadas. Com o tempo, há um aumento na densidade dos formigueiros em todas as classes de tamanho. $\mathrm{O}$ fato de a densidade de formigueiros diminuir quando a floresta está adulta pode estar relacionado ao fechamento do dossel da floresta, o que pode dificultar a instalação de novos formigueiros, ou pode estar relacionado à baixa diversidade do sub-bosque, ou seja, à carência de recursos vegetais para o forrageamento das formigas.

\section{Conclusões}

1. A densidade de formigueiros de Acromyrmex crassispinus varia sazonalmente e de acordo com a idade do Pinus taeda.

2. O tamanho dos formigueiros de Acromyrmex crassispinus aumenta com a idade do plantio de Pinus taeda.

\section{Agradecimentos}

À empresa Battistella Florestal S.A., pelo financiamento da pesquisa e cessão das áreas experimentais em Rio Negrinho, em especial aos engenheiros Ulisses Ribas e Reinaldo Langa, pelo apoio; à empresa Rigesa S.A., pelo financiamento da pesquisa e cessão das áreas experimentais em Três Barras; à Dra. Ana Paula Protti de Andrade Cruciol, da Universidade Estadual Paulista, Botucatu, pela identificação das espécies de formigas cortadeiras.

\section{Referências}

ALMEIDA, A.F. de; ALVES, J.E.M.; MENDES FILHO, J.M. de A.; LARANJEIRO, A.J. A avifauna e o sub-bosque como fatores auxiliares no controle biológico das saúvas em florestas implantadas. Silvicultura, v.8, p.145-150, 1983.

ARAÚJO, M. da S.; DELLA-LUCIA, T.M.C.; SOUZA, D.J. Estratégias alternativas de controle de formigas cortadeiras. Bahia Agrícola, v.6, p.71-74, 2003.

BENTO, J.M.S.; DELLA LUCIA, T.M.C.; MUCHOVEJ, R.M.C.; VILELA, E.F. Influência da composição química e da população microbiana de diferentes horizontes do solo no estabelecimento de sauveiros iniciais de Atta laevigata (Hymenoptera: Formicidae) em laboratório. Anais da Sociedade Entomológica do Brasil, v.20, p.307-317, 1991.

BUCHER, E.H.; MONTENEGRO, R. Hábitos forrajeros de cuatro hormigas simpátridas del gênero Acromyrmex (Hymenoptera: Formicidae). Ecología, v.2, p.47-53, 1974.

CANTARELLI, E.B. Silvicultura de precisão no monitoramento e controle de formigas cortadeiras em plantios de Pinus. 2005. 108p. Tese (Doutorado) - Universidade Federal de Santa Maria, Santa Maria.

CLARK, P.J.; EVANS, F.C. On some aspects of spatial pattern in biological populations. Science, v.121, p.397-398, 1955.

DIEHL-FLEIG, E.; ROCHA, E.S. da. Escolha do solo por fêmeas de Acromyrmex striatus (Roger) (Hymenoptera: Formicidae) para construção do ninho. Anais da Sociedade Entomológica do Brasil, v.27, p.41-45, 1998.

FORTI, L.C.; ANDRADE, M.L. de; ANDRADE, A.P.P.; LOPES, J.F.S.; RAMOS, V.M. Bionomics and identification of Acromyrmex (Hymenoptera: Formicidae) through an illustrated key. Sociobiology, v.48, p.135-153, 2006.

GONÇALVES, C.R. Ogênero Acromyrmex no Brasil(Hymenoptera: Formicidae). Studia Entomologica, v.4, p.113-180, 1961.

HERNÁNDEZ, J.V.; JAFFÉ, K. Dano econômico causado por populações de formigas Atta laevigata (F. Smith) em plantações de Pinus caribaea Mor. e elementos para o manejo da praga. Anais da Sociedade Entomológica do Brasil, v.24, p.287-298, 1995.

LIMA, C.A.; DELLA LÚCIA, T.M.C.; ANJOS, N.S. Formigas cortadeiras: biologia e controle. Viçosa: UFV, 2001. 28p. (Boletim de extensão, 44). 
LINK, D.; LINK, F.M.; OLIVEIRA, A.A. de. Potencial de dano da formiga preta, Acromyrmex crassispinus, em mudas de eucalipto e de pinus. In: SIMPÓSIO LATINO-AMERICANO SOBRE MANEJO FlORESTAL, 2., 2001, Santa Maria. Anais. Santa Maria: Universidade Federal de Santa Maria, 2001. p.671-676.

LINK, H.M.; LINK, F.M.; LINK, D. Controle da formiga-preta-pastadeira, Acromyrmex crassispinus, com formicidas em pó. Ciência Florestal, v.10, p.45-56, 2000.

LOPES, J.F.S.; FORTI, L.C.; CAMARGO, R.S. The influence of the scout upon the decision-making process of recruited workers in three Acromyrmex species (Formicidae: Attini). Behavioural Processes, v.67, p.471-476, 2004.

MAYHÉ-NUNES, A.J. Estudo de Acromyrmex (Hymenoptera: Formicidae) com ocorrência constatada no Brasil: subsídios para uma análise filogenética. 1991. 122p. Dissertação (Mestrado) - Universidade Federal de Viçosa, Viçosa.

PEREIRA, R. de C.; DELLA LUCIA, T.M.C.; MAYHÉ-NUNES, A.J. Levantamento de Attini (Hymenoptera: Formicidae) em povoamentos de Eucalyptus grandis W. Hill ex Maiden em Minas Gerais. Revista Árvore, v.23, p.341-349, 1999.

RANDO, J.S.S.; FORTI, L.C. Ocorrência de formigas Acromyrmex Mayr, 1865, em alguns municípios do Brasil. Acta Scientiarum. Biological Sciences, v.27, p.129-133, 2005.

REIS FILHO, W.; OLIVEIRA, S. de. Atividade externa, carregamento de isca granulada e controle de Acromyrmex crassispinus em floresta de Pinus taeda. Colombo: Embrapa Florestas, 2002. 3p. (Comunicado Técnico, 78).

SOCIEDADE BRASILEIRA DE SILVICULTURA. Fatos e números do Brasil florestal. São Paulo: SBS, 2006. 109p.

ZANETTI, R.; VILELA, E.F.; ZANÚNCIO, J.C.; LEITE, H.G.; FREITAS, G.D. Influência da espécie cultivada e da vegetação nativa circundante na densidade de sauveiros em eucaliptais. Pesquisa Agropecuária Brasileira, v.35, p.1911-1918, 2000.

Recebido em 15 de outubro de 2008 e aprovado em 31 de março de 2009 